\title{
OLHAR EMPREENDEDOR: FOMENTANDO EMPREENDEDORISMO E INOVAÇÃO EM UNIVERSIDADES
}

Nathalya Albina da Silva Pereira (nathalya.albina@gmail.com) - Universidade Estadual do Paraná, UNESPAR.

Maria Cecília Ferrari de Carvalho Teixeira (cecilia.ferraricarvalho@gmail.com) - Universidade Estadual do Paraná, UNESPAR.

Fernando Henrique Lermen (fernando.lermen@unespar.edu.br) - Universidade Estadual do Paraná, UNESPAR.

Roselis Natalina Mazzuchetti (roselis.mazzuchetti@unespar.edu.br) - Universidade Estadual do Paraná, UNESPAR.

\section{RESUMO}

Inovação e empreendedorismo são fatores que possuem a capacidade de potencializar e impulsionar um país. Uma abordagem para gerenciar esses fatores são os ecossistemas de inovação, que funcionam apenas com abordagens de inovação como Lean Startup e Design Thinking. O fomento da universidade empreendedora é essencial para a resolução de problemas da sociedade a partir do desenvolvimento de soluções inovadoras e sustentáveis. Neste contexto, este estudo objetiva apresentar um evento piloto denominado 'Olhar Empreendedor' para disseminação de empreendedorismo e inovação em universidades paranaenses. Para isto, este estudo empírico emprega entrevistas estruturadas utilizando Alpha de Cronbach para avaliar a consistência, além de estatística descritiva e análise de conteúdo. Como principais resultados ressalta-se o desenvolvimento de dois produtos, dois serviços e dois aplicativos voltados à resolução de problemas da sociedade e demais stakeholders. Como contribuição teórica têm-se o uso de metodologias para desenvolvimento de ofertas inovadoras e como práticas têm-se uma ação de extensão que promove pensamento empreendedor em diversos níveis de formação.

Palavras-chave: Universidade empreendedora; Inovação; Lean Startup; Design Thinking; Prototipagem. 


\section{INTRODUÇÃO}

Inovação e empreendedorismo são fatores que possuem a capacidade de potencializar e impulsionar um país (Quinn, 1979; Piñero-Chousa et al., 2020; Chakrabarty et al., 2021). Para que esses fatores funcionem, torna-se necessário projetar uma sequência de etapas em formato de projeto, contudo essa transformação demanda investimento, seja ele para o desenvolvimento da própria inovação ou para a obtenção do conhecimento necessário para isso (Di Vaio et al., 2021; Kreiser et al., 2021). Não obstante, ressalta-se a importância da criação de um ambiente oportuno que viabilize o projeto, que seja capaz de compartilhar conhecimento entre parceiros, sejam eles universidades, empresas e governo (Leonidou et al., 2017; Randhawa et al., 2021).

"Um ecossistema de inovação é o conjunto em evolução de atores, atividades e artefatos, e as instituições e relações, incluindo relações complementares e substitutas, que são importantes para o desempenho inovador de um ator ou de uma população de atores" (Granstrand and Holgersson, 2021, p. 3). Por outro lado, Rubens (2014) corrobora que um ecossistema de inovação vital é caracterizado por um alinhamento contínuo de relações sinérgicas que promovem o crescimento harmonioso do sistema em capacidade de resposta ágil às mudanças de forças internas e externas.

O fomento à ecossistemas de inovação ocorre apenas quando estruturado a partir de metodologias de desenvolvimento de produtos e serviços ágeis, buscando compreender o que é valorizado por diferentes stakeholders (Silva et al., 2020; Peralta et al., 2020). De acordo com Buchanan (1992), o Design Thinking é uma abordagem baseada em experimentos com equipes multidisciplinares e visa resolver problemas complexos e gerar soluções inovadoras por meio de uma etapa de identificação de problemas. Por outro lado, Ries (2011) relata que o Lean Startup é uma abordagem baseada em soluções visando testar empiricamente hipóteses que buscam a criação de um mínimo produto viável que atenda uma demanda real dos clientes.

No intuito de desenvolver um ecossistema de inovação a partir dessas abordagens, este estudo objetiva apresentar um evento piloto denominado 'Olhar Empreendedor' para disseminação de empreendedorismo e inovação em universidades paranaenses. Este estudo possui uma díade de contribuições; como contribuição teórica ressalta-se o uso de metodologias para desenvolvimento de ofertas inovadoras e como práticas têm-se uma ação de extensão que promove pensamento empreendedor em diversos níveis de formação. 


\section{METODOLOGIA}

Este estudo emprega uma abordagem de método misto (Venkatesh et al., 2013), empregando metodologias qualitativas (entrevistas semiestruturadas) e quantitativas (questionário de satisfação). Além de empregar metodologias de inovação como Lean Startup e Design Thinking. A Figura 1 apresenta a sequência metodológica deste estudo.

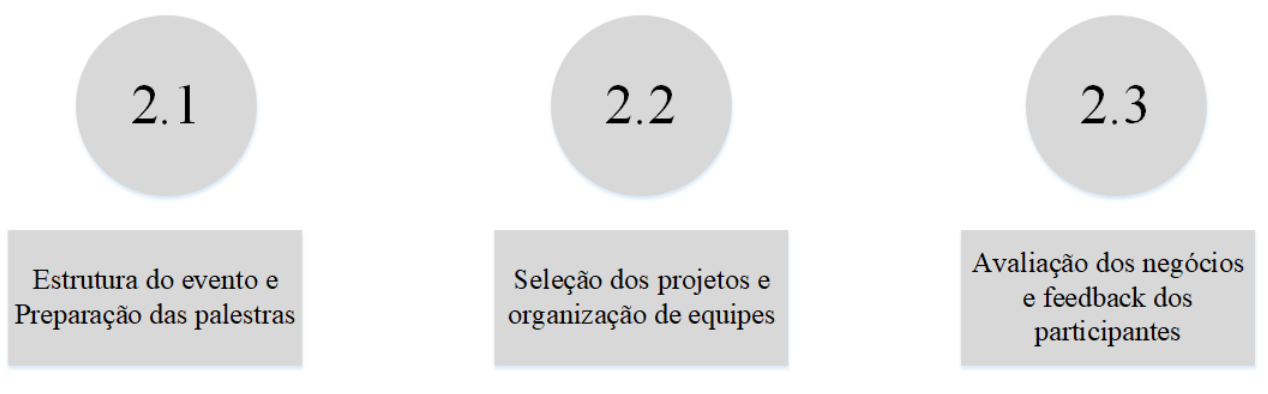

FIGURA 1 - Sequência Metodológica.

\subsection{Estrutura do evento e preparação de palestras}

O evento piloto "Olhar Empreendedor: Transformando ideias em futuros negócios" surgiu do desejo de professores de compartilhar conhecimentos oriundos do empreendedorismo e inovação para estudantes de graduação com potencial interesse em tornarem-se empreendedores da comunidade interna e externa da universidade. $\mathrm{O}$ foco inicial do evento foi o compartilhamento de diferentes metodologias e conhecimentos sobre o que envolve o empreendedorismo e sua aplicação, além de ter como propósito a realização do mesmo em outras universidades.

Na primeira reunião virtual do evento, ocorrida em março de 2021, com a participação de dois professores e duas bolsistas de extensão, foram definidos os objetivos, cursos, palestrantes, período de duração e aplicações das aulas/palestras ministradas que aconteceriam no decorrer do evento. Com base nisso, foram delegadas funções para cada membro da comissão organizadora, bem como, responsabilidades e atividades para a execução do evento. A divulgação teve início em abril de 2021 por meio de mídias sociais como facebook e instagram, além do site oficial (https://engdeproducaounespar.wixsite.com/my-site) com o regulamento/edital e horário das aulas, foram disponibilizados formulários de inscrição de projetos e equipes.

O conteúdo das aulas foi idealizado com o intuito de embasar o desenvolvimento de ofertas inovadoras e sustentáveis que se constituíssem em forma de produto, serviço, processo, Sistema Produto-Serviço (PSS), aplicativo ou software, assim possibilitando, através das metodologias aplicadas, criar um protótipo hipotético funcional. Essas metodologias abrangeram ferramentas específicas do empreendedorismo e inovação, ou seja, ferramentas 
viáveis para o desenvolvimento de startups com base estruturada em hipóteses geradas pelos participantes. Para a realização e consolidação dessa estrutura, técnicas como Lean Canvas, Mapa de Stakeholders, Value Proposition Canvas, Mínimo Produto Viável (MVP), criação de Survey, Mapa de Empatia, Persona, 5 porquês e Pitch foram aplicadas.

Além disso, conceitos sobre análise estatística da coleta de dados, estudo da viabilidade econômico-financeira e propriedade intelectual foram disponibilizados aos participantes. Tais técnicas têm como objetivo definir em relação aos projetos submetidos os problemas existentes e melhores soluções, os consumidores ideais que devem ser atingidos, as partes interessadas no projeto, um modelo de negócio que contenha desde partes que interessam o público-alvo até o retorno de investimento para o empreendedor, e por fim, a protótipo do projeto inscrito com a pesquisa de mercado realizada sobre o mesmo, assim sendo possível analisar de maneira qualitativa ou quantitativa o seu potencial diante uma amostra específica da sociedade, concluindo a viabilidade do projeto.

O evento teve o apoio de ministrantes de diversas áreas de pesquisa, que compõem o projeto de extensão devido à expertise referente às metodologias supracitadas. No total foram dez professores entre mestres e doutores, sendo eles engenheiros, administradores, estatísticos e arquitetos, entre outros. Estes estão ligados às universidades como: Universidade Estadual do Paraná (UNESPAR); Universidade de São Paulo (USP); Universidade Federal do Rio Grande do Sul (UFRGS); Universidade Federal do Pampa (UNIPAMPA); Instituto Federal do Rio Grande do Sul (IFRS); e, Universidade Presbiteriana Mackenzie (Mackenzie). Além disso, duas bolsistas de extensão (graduandas da UNESPAR) também ministraram durante o evento.

\subsection{Seleção dos projetos e organização de equipes}

Os projetos foram selecionados a partir de um formulário online elaborado pelo Google Forms $^{\circledR}$ (https://forms.gle/WVbKnU9swANCGjFs7), com período de submissão de 10 até 23 de abril de 2021, na qual, as informações principais consistiam no tema, justificativa e objetivo do projeto, como também em comentários adicionais sobre o objeto de pesquisa visado pelo mesmo e o seu potencial diante do mercado, ou seja, seus alvos principais no mercado consumidor. Esses tópicos serviram de base para a seleção dos projetos que pudessem ter potencial inovador, social, econômico ou ambiental. A partir da submissão dos projetos, foi disponibilizado outro instrumento de coleta realizado no Google Forms ${ }^{\circledR}$ (https://forms.gle/wDBy8hcyAewwjrNu6), com período de inscrição de 13 de abril até 07 de maio de 2021, referente a organização das equipes. Nesse questionário o objetivo central foi definir a posição de cada participante do evento nas equipes que se adequam aos projetos 
previamente submetidos. Essa identificação foi obtida com base na formação dos interessados em participar do evento, no perfil ao qual o participante se auto reconheceu (observador, devaneador, organizador, entusiasta e comunicador), e por fim, no motivo que o levou a participar do evento. Durante o evento, após o tema ministrado pelos professores, os mesmos foram até os grupos dar suporte para a aplicação das atividades, além do suporte de outros professores.

\subsection{Avaliação dos negócios e feedback dos participantes}

Ao final da primeira etapa do evento, ocorrida no dia 8 de maio de 2021, foi oferecida uma palestra sobre uma exposição que cada equipe deveria fazer apresentando o progresso de cada projeto. Para isto, foram realizadas duas rodadas de pitches, ambas com três minutos de apresentação, a qual foi avaliada o potencial de inovação, de escala, de mercado e da equipe. $\mathrm{Na}$ primeira rodada, o pitch parcial deveria conter o problema do projeto, solução, concorrência, cenário e evolução do projeto, além de informações futuras sobre o desenvolvimento pretendido pela equipe com o projeto. A comissão avaliadora foi composta por seis integrantes, entre eles professores da UNESPAR e convidados externos da UFRGS, UNIPAMPA, Universidade Tecnológica Federal do Paraná (UTFPR) e Universidade Estadual do Paraná (UEM).

Durante a segunda etapa do evento 'Olhar Empreendedor', realizada no dia 29 de maio de 2021, houve a apresentação final dos pitches dos projetos inscritos no evento. Desta vez, apenas cinco equipes estiveram presentes, a sexta equipe não compareceu e foi desclassificada. Deste modo, os grupos apresentaram ao final do dia novamente um novo pitch, contendo todos os aspectos relevantes e importantes de cada proposta. A exposição dos projetos apresentada teve novamente duração de três minutos e foi avaliada, novamente, por seis avaliadores, sendo eles professores da UNESPAR e convidados externos da UFRGS, IFRS e Mackenzie.

Como conclusão, foram distribuídos quatro prêmios, em primeiro lugar o projeto inovador, seguido por três outras categorias: (i) Economicamente viável, (ii) Responsabilidade social e (iii) Menor impacto ambiental. Após o evento, para avaliar a satisfação dos participantes do evento, aplicou-se um questionário estruturado preparado no Google Forms ${ }^{\circledR}$ (https://forms.gle/V5hL4QeANuATqAbp9) com o objetivo de mensurar pontos fortes, pontos fracos e pontos a melhorar para próximos eventos. Com isso, obteve-se uma amostra de 22 respondentes, calculando assim, o Alpha de Cronbach para avaliar a confiabilidade e 
consistência do instrumento e entendimento dos participantes (Cronbach, 1951; Brown, 2002), conforme apresentado na Tabela 1.

TABELA 1 - Alpha de Cronbach do instrumento de feedback.

\begin{tabular}{|c|c|c|c|c|}
\hline Item & Alpha de Cronbach & Alpha Padrão & G6(smc) & R Médio \\
\hline Todas as questões do instrumento & 0,764 & 0.8 & 1 & 1,379 \\
\hline
\end{tabular}

O Alpha de Cronbach obtido pela aplicação do questionário de satisfação obteve o valor de 0,764, ou seja, este é superior ao valor mínimo aceitável de 0,70 (Taber, 2018). Portanto, a partir destes valores, conclui-se que os resultados são fundamentados. O alpha padrão é considerado o Alpha de Cronbach aperfeiçoado, pois realiza a correlação de todos os fatores separadamente, desta maneira verifica-se a maior consistência dos resultados obtidos (Duhachek et al., 2005). Não obstante, ressalta-se a identificação do G6(smc), que de acordo com literatura, quanto mais próximo de um, melhor (Revelle, 2013). Por fim, o R médio representa a média das médias de todos os elementos do formulário, contendo o valor de 1,379. Assim, verifica-se que o questionário aplicado para avaliar o feedback dos participantes foi considerado consistente e confiável.

Após a validação do instrumento, foram utilizadas estatísticas descritivas para mensurar as escolhas dos respondentes quanto à probabilidade de recomendação, qualidade do evento, nível de organização, qualidade dos organizadores e duração do evento. Além disso, empregou-se a análise de conteúdo (Elo \& Kyngäs, 2008) de perguntas abertas relacionadas aos pontos fortes e fracos do evento. Não obstante, o perfil do participante foi avaliado em relação ao interesse antes e depois do evento em abrir uma empresa, nível empreendedor, se adquiriu algumas competências e habilidades, e ainda, quais dessas habilidades adquiriram. Por fim, ressalta-se a informação de universidade, curso, gênero e idade dos participantes.

\section{RESULTADOS E DISCUSSÃO}

Esta seção é composta por quatro seções, tais como: (i) soluções desenvolvidas e pontuações dos pitches; (ii) perfil demográfico dos participantes; (iii) perfil empreendedor; e (iv) lições aprendidas - pontos fortes e fracos.

\subsection{Soluções desenvolvidas e pontuações dos pitches}

Durante a realização do evento foram criadas seis soluções que entregam valor para a sociedade, dentre elas estão: Grupo 1 - Aplicativo que trata relação do mecânico e motoristas; Grupo 2 - Bicicletas elétricas para a região portuária; Grupo 3 - Guia para ostreicultores e 
consumidores de ostras; Grupo 4 - Alicates ergonômicas para profissionais da beleza; Grupo 5

- Aplicativo para gestão financeira; Grupo 6 - Loja física voltada à experiência do usuário sustentável. Estes grupos participaram de duas rodadas de pitches (parcial e final) com quatro métricas que possuíam notas de 1 a 10, conforme apresentado na Tabela 2.

TABELA 2 - Pontuações de grupos nos pitches parcial e final.

\begin{tabular}{|c|c|c|c|c|c|c|c|}
\hline \multirow{4}{*}{ Pitch } & \multirow{2}{*}{ Potencial } & \multicolumn{6}{|c|}{ Grupos } \\
\cline { 3 - 8 } & & Grupo 1 & Grupo 2 & Grupo 3 & Grupo 4 & Grupo 5 & Grupo 6 \\
\hline \multirow{4}{*}{$\begin{array}{c}\text { Pitch } \\
\text { Parcial }\end{array}$} & Inovação & 9,5 & 7,8 & 8,2 & 8,8 & 9,5 & 8,9 \\
\cline { 2 - 8 } & Escala & 7,6 & 9,0 & 7,6 & 8,6 & 8,6 & 8,0 \\
\cline { 2 - 8 } & Mercado & 9,0 & 8,8 & 8,0 & 8,4 & 9,2 & 8,8 \\
\cline { 2 - 8 } & Equipe & 8,0 & 7,8 & 8,4 & 8,7 & 9,0 & 8,8 \\
\hline \multirow{4}{*}{$\begin{array}{c}\text { Pitch } \\
\text { Final }\end{array}$} & Inovação & 9,0 & 7,0 & 0,0 & 8,0 & 9,0 & 9,0 \\
\cline { 2 - 8 } & Escala & 10,0 & 6,0 & 0,0 & 9,0 & 10,0 & 9,0 \\
\cline { 2 - 8 } & Mercado & 10,0 & 8,0 & 0,0 & 9,0 & 9,0 & 8,0 \\
\cline { 2 - 8 } & Equipe & 8,0 & 7,0 & 0,0 & 9,0 & 9,0 & 8,0 \\
\hline \multicolumn{2}{|c|}{ Média total } & 8,89 & 7,68 & 4,03 & 8,69 & 9,16 & 8,56 \\
\hline
\end{tabular}

Contudo, ressalta-se que o Grupo 5 recebeu o prêmio de projeto inovação e os Grupos 1, 4 e 6 receberam os prêmios economicamente viáveis, responsabilidade social e menor impacto ambiental, respectivamente. Nota-se que a existência de um prêmio fez os participantes se engajarem mais no evento (Manzini \& Vezzoli, 2003), além disso, é notado que o Grupo 3 não pôde participar no pitch final.

\subsection{Perfil demográfico dos participantes}

Para compreender o perfil dos 22 participantes do evento que compõem a amostra, mapeou-se a partir da Tabela 3 as informações coletadas no instrumento.

TABELA 3 - Perfil demográfico da amostra.

\begin{tabular}{|c|c|c|c|c|c|}
\hline Município & Quantidade & $(\boldsymbol{\%})$ & Universidade & Quantidade & $(\boldsymbol{\%})$ \\
\hline Paranaguá & 19 & $86,4 \%$ & UNESPAR & 19 & $86,4 \%$ \\
\hline Ponta Grossa & 2 & $9,1 \%$ & UTFPR & 2 & $9,1 \%$ \\
\hline Maringá & 1 & $4,5 \%$ & UEM & 1 & $4,5 \%$ \\
\hline Idade (anos) & Quantidade & $(\boldsymbol{\%})$ & Gênero & Quantidade & $(\boldsymbol{\%})$ \\
\hline Entre 18 e 25 & 17 & $77,3 \%$ & Masculino & 10 & $45,4 \%$ \\
\hline
\end{tabular}




\begin{tabular}{|c|c|c|c|c|c|}
\hline Entre 26 e 35 & 3 & $13,6 \%$ & Feminino & 11 & $50 \%$ \\
\hline Acima de 36 & 2 & $9,0 \%$ & Não informa & 1 & $4,5 \%$ \\
\hline
\end{tabular}

Ressalta-se que a maioria é proveniente da UNESPAR e do município de Paranaguá, localização na qual o evento foi realizado. Ainda, conclui-se que a faixa etária da maioria dos respondentes é entre 18 e 25 anos, podendo notar que pessoas mais jovens possuem maior interesse em atividades empreendedoras (Henderson \& Robertson, 2000). Por fim, nota-se que o gênero não foi um fator notável, por possuir estabilidade entre os participantes.

\subsection{Perfil empreendedor dos participantes}

Dentre as questões do instrumento para avaliar o feedback dos participantes, foram levantadas informações quanto ao perfil empreendedor e interesse em criar uma startup. Na literatura, quanto ao perfil, competências e características de um empreendedor, percebeu-se uma ampla categoria de análise devido à vasta quantidade de atributos (por exemplo, Ray, 1993; Jusoh et al., 2013; Abdullah et al., 2018). Desta maneira, definiram-se a adesão das características empreendedoras com base nas competências elencadas por Dornelas (2015, p. 23-24).

Os participantes do 'Olhar Empreendedor' relataram que, após o evento, adquiriram a habilidade de tomarem melhores decisões $(81,9 \%)$ e, se declararam, mais dinâmicos $(72,7 \%)$, ou seja, não se sentem inseguros ao lidar com adversidades e eliminam barreiras com facilidade, fator importante na área de empreendedorismo (Sandhu et al., 2011). Não obstante, $59,1 \%$ dos participantes revelaram que adquiriram novos conhecimentos no evento e se tornaram mais determinados $(54,5 \%)$, logo, comprova-se que o evento é capaz de aumentar a expertise dos integrantes, além de torná-los perseverantes frente aos seus objetivos.

Os dados afirmam que 54,5\% dos respondentes acreditam que são capazes de criar valor para a sociedade, estabelecendo medidas e estratégias que geram retorno positivo ambiental e social. Nota-se que parte dos participantes afirmam adquirir a competência de ser visionário (41\%) ao participarem do evento, revelando que possuem uma maior visão de futuro para o negócio em que estão envolvidos (Taylor et al., 2004).

Apenas sete participantes relataram acreditar que se sentem mais independentes $(31,8 \%)$, ou seja, que são capazes de assumir os riscos não calculados e calculados (31,8\%). Ressalta-se que estes dados são referentes às características empreendedoras que os participantes acreditam terem conquistado após o evento, portanto, não se sabe ao certo se os participantes já possuíam essas características ou se apenas, não as alcançaram (Sousa, 2018). Entre os participantes, evidenciou-se que após o 'Olhar Empreendedor', 27,3\% acreditaram se tornar 
indivíduos mais organizados e que $22,7 \%$ desenvolveram fatores relacionados ao otimismo e a capacidade de criar um vínculo afetuoso com seus respectivos projetos.

Com relação ao interesse na criação de uma startup, 63,6\% dos participantes relataram ter o desejo de montar um negócio antes do evento, e 72,7\% manifestou pretender abrir uma empresa após o 'Olhar Empreendedor'. Logo, percebe-se que houve um aumento de 9,1\% na pretensão de abrir uma empresa após o evento, confirmando o relatado por Giudici (2019) que eventos de empreendedorismo fortalecem a intenção de criação de startups.

Perante os dados levantados, é nítido que todos os participantes adquiriram novas características empreendedoras, comprovando que o evento é capaz de desenvolver o pensamento crítico e que o movimento universidade empreendedora é necessário. Contudo, os participantes do evento mostraram-se interessados em desenvolver produtos, serviços e aplicativos na prática, desta maneira, se faz necessária uma avaliação futura com estes mesmos respondentes, para verificar quais das seis equipes efetivaram seus projetos e obtiveram sucesso no empreendimento.

\subsection{Lições aprendidas: Pontos fortes e fracos}

Quanto às questões abertas do instrumento, 13 dos 22 participantes relataram que as palestras foram de grande contribuição empreendedora, em especial, ressalta-se a fala do R13 “Método excelente de ensino de todos os docentes e palestrantes, templates organizados e bem-feitos, tudo foi incrível!", e ainda, o mencionado pelo R21 "As palestras têm alto grau de aplicabilidade em empreendimentos inovadores e que ainda não se inseriram no mercado". Além de comentarem os respondentes R4, R5, R12 e R14 corroboraram que a participação de professores e palestrantes de várias áreas foi de grande valia para agregar conhecimento diverso. Ainda sobre a avaliação das palestras, os respondentes 20 dos 22 participantes manifestaram que as acharam dinâmicas, com conteúdo enriquecedor e muito claras.

Acerca dos pontos fracos do evento, 16 dos 22 participantes relataram problemas quanto à longa duração e dias do evento, por ocorrer num sábado, além de mencionarem também os respondentes R1 e R19 o curto prazo de aplicação das atividades práticas. Ainda neste contexto, os respondentes R3 e R10 expressaram descontentamento com o tempo de aplicação das metodologias, contexto pouco declarado na literatura de empreendedorismo (Raeesi et al., 2013).

Quanto a pontos de melhorias, 14 dos 22 respondentes sugeriram a diminuição de carga horária ou distribuição do evento em mais dias, como também a ocorrência do mesmo em dias de semana, além do R4 aconselhar uma maior divulgação sobre o evento. A Figura 2 
apresenta o cronograma do evento realizado que possui os problemas mencionados pelos participantes do evento.

\section{Cronograma}

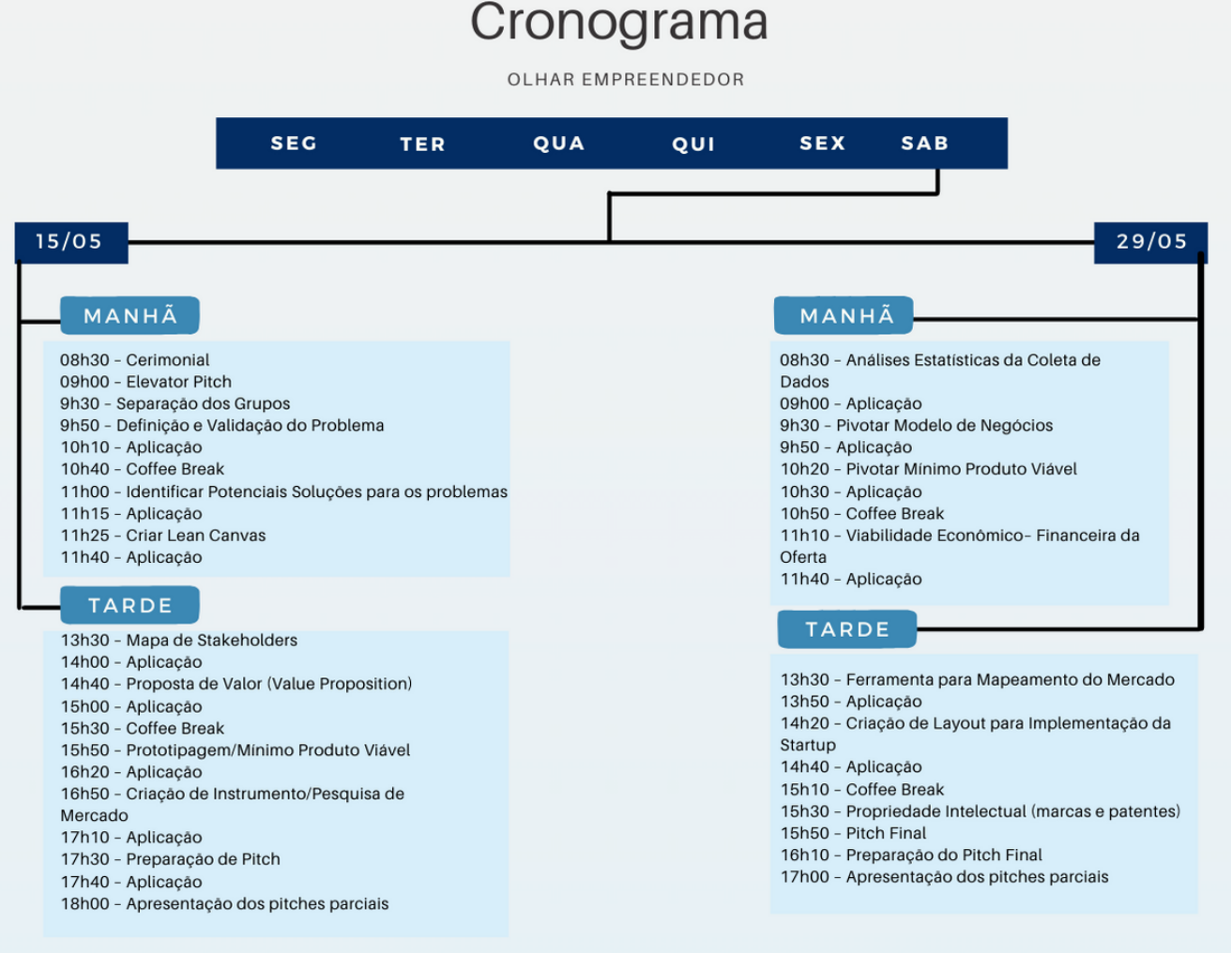

FIGURA 2 - Cronograma do evento Olhar Empreendedor

Com as informações obtidas na pesquisa de satisfação sobre pontos fracos, fortes e possíveis melhorias, o horário e disposição das aulas e aplicações foram reavaliados. A Figura 3 apresenta a melhor forma para o desenvolvimento de uma próxima edição.

IDENTIFICAR POTENCIAIS SOLUCOOES PARA OS PROBLEMAS ( $40 M I N$ AULA E APLICACCAOO)

LEAN CANVAS (50MIN AULA E APLICACÃO)

PROPOSTA DE VALOR (50MIN AULA E APLICACCAO)

MAPA DE STAKEHOLDERS (50MIN AULA E APLICACĀO) MAPEAMENTO DO MERCADO (50MIN AULA E APLICACAO) MVP (5OMIN AULA E APLICAÇÁO)

VIABILIDADE ECONOMICO-FINANCEIRA (50MIN AULA APLICAÇÃO)

INSTRUMENTO DE MERCADO (50MIN AULA E APLICAC̄ĀO)

ANALISE ESTATISTICA (4OMIN AULA)

PITCH PARCIAL (1OMIN AULA E APLICAÇÃO)

$\stackrel{\circ}{\subseteq}$

$\underset{\mathrm{m}}{\mathrm{m}}$
DIAS DO EVENTO

DIAS PARA A COLETA DE DADOS
ํㅗㅁ

$\stackrel{\text { o }}{>}$

\section{Cronograma}

OLHAR EMPREENDEDOR

ANALISE ESTATISTICA (4OMIN AULA E APLICACẢO) PIVOTAR MVP (50MIN AULA E APLICAÇẢO) PIVOTAR LEAN CANVAS (5OMIN AULA E APLICAÇẢO)

PITCH FINAL (40MIN AULA E APLICACẢO)

APRESENTAÇOES

PREMIAÇÃO 
FIGURA 3 - Cronograma ajustado do evento Olhar Empreendedor

Nota-se que com as alterações propostas no evento, os futuros participantes possuirão tempo maior para a coleta de dados e os dias de evento não possuem mais que três horas de duração. Mesmo assim, este novo cronograma atenderia aos participantes da edição piloto e suporta acadêmicos e práticos que visam a implementação de evento de empreendedorismo em empresas e universidades.

\section{CONCLUSÃO}

Este estudo objetivou apresentar um evento piloto denominado 'Olhar Empreendedor' para disseminação de empreendedorismo e inovação em universidades paranaenses. Para isto, este estudo empírico emprega entrevistas estruturadas utilizando Alpha de Cronbach para avaliar a consistência, além de estatística descritiva e análise de conteúdo.

Como principais resultados ressalta-se o desenvolvimento de dois produtos, dois serviços e dois aplicativos voltados à resolução de problemas da sociedade e demais stakeholders. Como contribuição teórica têm-se o uso de metodologias para desenvolvimento de ofertas inovadoras e como práticas têm-se uma ação de extensão que promove pensamento empreendedor em diversos níveis de formação.

Como limitação, tem-se o tamanho da amostra limitado devido a ser um evento piloto e a falta dos participantes do grupo 3 na segunda semana. Contudo, como futuras pesquisas, sugere-se o desenvolvimento de novos estudos voltados ao empreendedorismo em universidades e empresas brasileiras para o desenvolvimento de soluções que atendam as demandas de diversos stakeholders, assim como, realizar dinâmicas para o fomento à universidade empreendedora.

\section{REFERÊNCIAS}

ABDULLAH, Naziruddin; HADI, Noor Ul; DANA, Léo-Paul. The nexus between entrepreneur skills and successful business: a decompositional analysis. International Journal of Entrepreneurship and Small Business, v. 34, n. 2, p. 249-265, 2018.

BROWN, James Dean. The Cronbach alpha reliability estimate. JALT Testing \& Evaluation SIG Newsletter, v. 6, n. 1, 2002.

BUCHANAN, Richard. Wicked problems in design thinking. Design issues, v. 8, n. 2, p. 5-21, 1992.

CHAKRABARTY, Arindam; NORBU, Tenzing. Innovation, Entrepreneurship and Sustainability of Business Through Techno-Social Ecosystem-Indian Scene. In: Research in Intelligent and Computing in Engineering. Springer, Singapore, 2021. p. 131-139.

CRONBACH, Lee J. Coefficient alpha and the internal structure of tests. Psychometrika, v. 16, n. 3, p. 297334, 1951.

DA LUZ PERALTA, Carla Beatriz et al. A framework proposition to identify customer value through lean practices. Journal of Manufacturing Technology Management, 2020. 
DI VAIO, Assunta et al. The role of digital innovation in knowledge management systems: A systematic literature review. Journal of Business Research, v. 123, p. 220-231, 2021.

DORNELAS, J. C. A. Empreendedorismo corporativo: como ser empreendedor, inovar e se diferenciar na sua empresa. Rio de Janeiro: Elsevier, 2008.

DUHACHEK, Adam; COUGHLAN, Anne T.; IACOBUCCI, Dawn. Results on the standard error of the coefficient alpha index of reliability. Marketing Science, v. 24, n. 2, p. 294-301, 2005.

ELO, Satu; KYNGÄS, Helvi. The qualitative content analysis process. Journal of Advanced Nursing, v. 62, n. 1, p. 107-115, 2008.

HENDERSON, Roger; ROBERTSON, Martyn. Who wants to be an entrepreneur? Young adult attitudes to entrepreneurship as a career. Career Development International, v. 5, n. 6, p. 279-287, 2000.

JUSOH, Rosnani et al. Entrepreneur training needs analysis: Implications on the entrepreneurial skills needed for successful entrepreneurs. International Business \& Economics Research Journal (IBER), v. 10, n. 1, 2011.

KREISER, Patrick M. et al. Corporate entrepreneurship strategy: extending our knowledge boundaries through configuration theory. Small Business Economics, v. 56, n. 2, p. 739-758, 2021.

LEONIDOU, Erasmia et al. An integrative framework of stakeholder engagement for innovation management and entrepreneurship development. Journal of Business Research, 2018.

MANZINI, Ezio; VEZZOLI, Carlo. A strategic design approach to develop sustainable product service systems: examples taken from the 'environmentally friendly innovation'Italian prize. Journal of Cleaner Production, v. 11, n. 8, p. 851-857, 2003.

PIÑEIRO-CHOUSA, Juan et al. Innovation, entrepreneurship and knowledge in the business scientific field: Mapping the research front. Journal of Business Research, v. 115, p. 475-485, 2020.

QUINN, James Brian. Technological innovation, entrepreneurship, and strategy. Sloan Management Review (pre-1986), v. 20, n. 3, p. 19, 1979.

RAEESI, Ramin et al. Understanding the interactions among the barriers to entrepreneurship using interpretive structural modeling. International Journal of Business and Management, v. 8, n. 13, p. 56, 2013.

RANDHAWA, Krithika et al. Evolving a Value Chain to an Open Innovation Ecosystem: Cognitive Engagement of Stakeholders in Customizing Medical Implants. California Management Review, v. 63, n. 2, p. 101-134, 2021.

RAY, Dennis M. Understanding the entrepreneur: entrepreneurial attributes, experience and skills. Entrepreneurship \& Regional Development, v. 5, n. 4, p. 345-358, 1993.

RIES, Eric. The lean startup: How today's entrepreneurs use continuous innovation to create radically successful businesses. Currency, 2011.

SANDHU, Manjit Singh; SIDIQUE, Shaufique Fahmi; RIAZ, Shoaib. Entrepreneurship barriers and entrepreneurial inclination among Malaysian postgraduate students. International Journal of Entrepreneurial Behavior \& Research, 2011.

SILVA, Diego Souza et al. Lean Startup, Agile Methodologies and Customer Development for business model innovation: A systematic review and research agenda. International Journal of Entrepreneurial Behavior \& Research, 2020.

SOUSA, Maria José. Entrepreneurship skills development in higher education courses for teams leaders. Administrative Sciences, v. 8, n. 2, p. 18, 2018.

TABER, Keith S. The use of Cronbach's alpha when developing and reporting research instruments in science education. Research in Science Education, v. 48, n. 6, p. 1273-1296, 2018.

TAYLOR, David W.; WALLEY, Elizabeth E. The green entrepreneur: opportunist, maverick or visionary?. International Journal of Entrepreneurship and Small Business, v. 1, n. 1-2, p. 56-69, 2004.

VENKATESH, Viswanath; BROWN, Susan A.; BALA, Hillol. Bridging the qualitative-quantitative divide: Guidelines for conducting mixed methods research in information systems. MIS quarterly, p. 21-54, 2013. 